\title{
Inhalt des LV. Bandes
}

\section{Romanistische Abteilung.}

Seite

Otto Lenel † von Leopold Wenger . . . . . . . . . VII

Beseler, Gerhard, Zur Lehre von der Präterition . . . . . I

Düll, Rudolf, Vom vindex zum iudex (Fortsetzung und Schluß) 9

Ehrhardt, Arnold, Litis aestimatio in der Zeit vor den leges Iuliae iudiciariae . . . . . . . . . . . . . 36

Kreller, Hans, Zur Frage der Zuverlässigkeit unserer Gaiusüberlieferung. . . . . . . . . . . . . 159

Schönbauer, Ernst, Vom Bodenrecht zum Bergrecht. Studien zur Geschichte des Bergbaurechts .......... 183

Siber, Hein rich, Cäsars Diktatur und das Prinzipat des Augustus 99

\section{Miszellen:}

Balis, Georg, Die Natur der actio suppletoria . . . . . . 272

Beseler, Gerhard, Bindung und Lösung. . . . . . . . 226

Cvetler, Jiř́, Daneion und Chresis . . . . . . . . 276

Genzmer, Erich, Seckel und Ugo Nicolini über die Quaestionen des Pillius . . . . . . . . . . . . . . . . 315 mit zwei Anhängen aus Seckels Nachlaß:

Seckel, Emil †, Die Casus Bambergenses . . . . . . . . 336

—, -, Die Quaestiones Vindobonenses des Johannes Bassianus . . . . . . . . . . . . . . 338

Kretschmar, Paul, Zur Zahlensymbolik in der mittelalterlichen Digesten-Einteilung. . . . . . . . . . 308

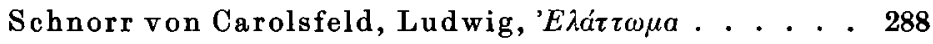

Taubenschlag, Rafael, Der Schutz der Rechtsverhältnisse an Liegenschaften im gräko-ägyptischen Recht . . . . 278

Wieacker, Franz, Die Struktur der Sabinusmasse . . . . . 292

\section{Literatur:}

Alt, Albrecht, Die Ursprünge des israelitischen Rechts. . 359 Besprochen von Artur Weiser.

Arangio-Ruiz, Vincenzo, Cours de droit romain . . . . 479 Kurz angezeigt von $H$ ans $K r e l l e r$. 
Archi, Gian Gualberto, Il trasferimento della proprietd nella compravendita romana . . . . . . . . 395 Besprochen von Max $\mathrm{Kaser}$.

Bassanelli, Enrico, La Colonia perpetua . . . . . . 453 Besprochen von Heinrich Erman.

Berve, Helmut, Griechische Geschichte II . . . . . . 365 Besprochen von Walther Kolbe.

Betti, Emilio, Corso di istituzioni di diritto romano II. . . . Kurz angezeigt von Hans $\mathrm{K}$ reller.

Bibliotheca philologica classica 59, bearbeitet von Wilhelm Rechnitz................ . 480 Kurz angezeigt von Hans Kreller.

Bozza, Francesco, Il matrimonio nel diritto attico... . -, -, Il matrimonio nel diritto dei papiri dell' epoca tolemaica Kurz angezeigt von $\mathrm{Hans} \mathrm{K}$ reller.

Callegari, Dante, L'ipoteca mineraria . . . . . . . 457 Besprochen von Hans Njedermeyer.

Cavin, Pierre-Eugène, L'extinction de l'usufruit ,rei mutatione“................. . 399 Besprochen von Erich Berneker.

Chiazzese, Lauro, Confronti testuali, Parte generale . . 443 Besprochen von Bernhard $K \ddot{\text { übler. }}$

Dulckeit, Gerhard, Erblasserwille und Erwerbswille bei Antretung der Erbschaft ............. 403 Besprochen von Ulrich v. Lübtow.

Erdmann, Walter, Die Ehe im alten Griechenland . . . 363 Besprochen von Hans Kreller.

Frezza, Paolo, Osservazioni sopra il sistema di Sabino . . 388 Besprochen von Hugo Krüger.

Grohmann, Adolf, Arabische Papyri aus den staatlichen Museen zu Berlin, Band I, Teil I . . . . . . . . . .

—, -, Arabic Papyri in the Egyptian Library, Vol. I . . . . 459 Besprochen von Marian San Nicolò.

Hedemann, Justus Wilhelm, Die Fortschritte des Zivilrechts im XIX. Jahrhundert, II. Teil, 2. Hälfte . . . 475 Besprochen von Hans Kreller.

Hellebrand, Walter, Das ProzeBzeugnis im Rechte der gräko-ägyptischen Papyri, I. Teil . . . . . . . . . . 372 Besprochen von Max Kaser.

Hill, George, Treasure trove in the law and practice of antiquity ................... . 452 Besprochen von Georg EiBer. 
Longo, Giannetto, L'hereditatis petitio . . . . . . . 413 Besprochen von Georg H. Maier.

Marsh, Frank Burr, The Reign of Tiberius . . . . . . 380 Besprochen von Heinrich Siber.

Mazeaud, Jean, La Nomination du Iudex unus sous la procédure formulaire à Rome . . . . . . . . . . . . . 424 Besprochen von Leopold Wenger.

Momigliano, Arnaldo, Claudius, The Emperor and his achievement, translated by W. D. Hogarth . . . . . 385 Besprochen von Heinrich Siber.

Monier, Ray mond, Manuel élémentaire de droit romain, Tome premier............... Kurz angezeigt von Hans Kreller.

Monti, Gennaro Maria, Le corporazioni nell' evo antico e nell' Alto Medio Evo . . . . . . . . . . . . . . . Besprochen von Ludwig Schnorr v. Carolsfeld.

Moschetti, Guiscardo, Tre documenti Veronesi dei Secoli XI e XII e la „Lex Romana canonice compta“ . . . . 464 Besprochen von Erich Genzmer.

Papyri und Altertumswissenschaft, Vorträge des dritten internationalen Papyrologentages in München, herausgeg. von Walter Otto und Leopold Wenger. . . . . . Besprochen von Otto Eger.

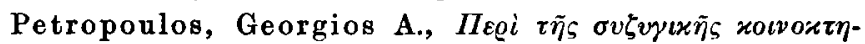

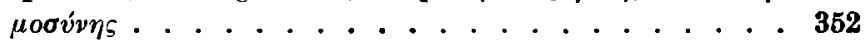
Besprochen von Erwin Seidl.

Pirenne, Jacques, Histoire des institutions et du droit privé de l'ancienne Egypte, I. II . . . . . . . . . . . 345 Besprochen von Erwin Sefdl.

Pufendorf, Samuel, De Iure Naturae et Gentium Libri Octo, I. II. . . . . . . . . . . . . . . . . . . 469 Besprochen von Julius Binder.

Rotondi, Mario, Dogmatica e diritto comparato . . . . 473 Besprochen von Hans Kreller.

Sanfilippo, Cesare, Il metus nei negozi giuridici . . . . 402 Besprochen von Georg H. Maier.

Simonius, August, Was bedeuten für uns die Pandekten ? 477 Besprochen von Heinrich Stoll.

Solazzi, Siro, La revoca degli atti fraudolenti nel diritto romano . . . . . . . . . . . . . . 419 Besprochen von Erich-Hans Kaden.

Sommer, Ferdinand, Die Ab̧hijavā-Urkunden . . . . . 354 Besprochen von Paul Koschaker. 
Sommer, Ferdlinand, Aḥhijavāfrage und Sprachwissenschaft 358 Besprochen von Paul Koschaker.

Väth, Alfons, Die Inder . . . . . . . . . . . . 483 Kurz angezeigt von Hans Kreller.

Wahrmund, Ludwig, Das Institut der Ehe im Altertum . . 361 Besprochen von Georg EiBer.

Wlassak, Moriz, Konfessio in Iure und Defensionsverweigerung nach der Lex Rubria de Gallia Cisalpina . . . . 376

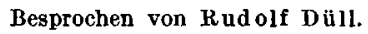

Weitere Büchereingänge........... 479

\section{Mitteilungen :}

Kurzer Bericht über die vierte Tagung deutscher Rechtshistoriker in Köln 1934 . . . . . . . . . . . . . . . 485 Erstattet von Franz Wieacker und Eugen Wohlhaupter.

Congressus Iuridicus Internationalis (Rom 1934) . . . . . 491 Bericht von Leopold Wenger.

Vierter Internationaler Papyrologentag. . . . . . . . . . 496

Zum 80. Geburtstage Moriz Wlassaks, Glückwunschadresse und Dankschreiben . . . . . . . . . . . . . . . . . 497

Aufruf der Preußischen Akademie der Wissenschaften, betr. Biographie Theodor Mommsens . . . . . . . 498

Förderung wissenschaftlicher Auslandsbeziehungen . . . . . 499

In memoriam . . . . . . . . . . . . . . . . . . . . . . 500 Anton vorı Premerstein $\dagger$ (Wilhelm EnBlin).

Alphabetisches Verzeichnis der Mitarbeiter andiesem Bande:

Balis S. 272. - Berneker S. 399. - Beseler S. 1. 226. - Binder S. 469. - Cretler S. 275. - Düll S. 9. 376. - Eger S. 368. - Ehrhardt S. 36. Eißer S. 361. 452. - Enßlin S. 500. - Erman S. 453. - Genzmer S. 315. 336. 338. 464. - Kaden S. 419. - Kaser. S. 372. 395. - Kolbe S. 365. - Koschaker S. 354. 358. - Kreller S. 159. 363. 473. 475.479. 496. - Kretschmar S. 308. - Krüger S. 388. - Kübler S. 443. v. Lübtow S. 403. - Maier S. 402. 413. - Niedermeyer S. 457. - San Nicolò S. 459. - Schnorr v. Carolsfeld S. 288. 449. - Schönbauer S. 183. - Seidl S. 345. 352. - Siber S. 99. 380. 385. - Stoll S. 477. Taubenschlag S. 278. - Weiser S. 359. - Wenger S. VII. 424. 491. Wieacker S. 292. 485. - Wohlhaupter S. 485. 